$\begin{array}{ll} & \text { Etnográfica } \\ \text { etnográfica } & \text { Revista do Centro em Rede de Investigação em }\end{array}$

Antropologia

vol. 14 (1) | 2010

Vol. $14(1)$

\title{
Trabalhos e registos
}

\section{Branca Moriés e Clara Saraiva}

URL: https://journals.openedition.org/etnografica/182

DOI: 10.4000/etnografica.182

ISSN: 2182-2891

\section{Editora}

Centro em Rede de Investigação em Antropologia

\section{Edição impressa}

Data de publição: 1 fevereiro 2010

Paginação: 177-184

ISSN: 0873-6561

\section{Refêrencia eletrónica}

Branca Moriés e Clara Saraiva, «Trabalhos e registos», Etnográfica [Online], vol. 14 (1) | 2010, posto online no dia 19 outubro 2011, consultado o 12 fevereiro 2022. URL: http://journals.openedition.org/ etnografica/182 ; DOI: https://doi.org/10.4000/etnografica.182

\section{(c) (†) \$}

Etnográfica is licensed under a Creative Commons Attribution-NonCommercial 4.0 International License. 


\section{Trabalhos e registos}

\section{Compilado por Branca Moriés \\ e Clara Saraiva}

\section{PRÉMIOS E DISTINÇÕES}

- Prémio Gomes Pereira, atribuído às obras Técnicas de Fiação Primitiva e Escultura Africana, 1968.

- Condecorado pelo presidente da República, Jorge Sampaio, com a Ordem de Santiago, pelos serviços prestados ao país em prol da cultura, 10 de Junho de 2001.

\section{LIVROS E ARTIGOS PUBLICADOS}

- “Notas etnográficas de Caíde”, Douro Litoral, 9. a série, III, 1959, pp. 57 1-584.

- "Subsídios para o estudo da fiação e tecelagem em Portugal", Trabalhos de Antropologia e Etnologia, XVIII (1-2), Porto, 1960-1961, pp. 141-148.

- "Assadas de batatas na areia", Trabalhos de Antropologia e Etnologia, XVIII (1-2), Porto, 1960-1961, pp. 155-156.

- "A sidra no Norte de Portugal", Trabalhos de Antropologia e Etnologia, XVIII (3-4), Porto, 1961-1962, pp. 362-366.

- "Subsídios para o estudo do leite e da manteiga no Norte de Portugal", Trabalhos de Antropologia e Etnologia, XVIII (3-4), Porto, 1961-1962, pp. 357-362.

- "Vestígios do culto das pedras no Norte de Portugal”, Actas do Congresso Luso-Espanhol para o Progresso das Ciências, secção VII, Porto, Associação Portuguesa para o Progresso das Ciências, 1962, pp. 1-7.

- "Aspectos tradicionais do leite e da manteiga", Gazeta das Aldeias, 2493, Alverca.

- Bibliografia Analítica da Etnografia Portuguesa, Lisboa, IAC, 1963, 670 pp.

- Moinhos de Vento: Açores e Porto Santo, Lisboa, IAC, 1965, 118 pp. (em colaboração com Ernesto Veiga de Oliveira e Fernando Galhano). 
- "Calçado de pau em Portugal", Revista de Etnografia, VII (I), Porto, 1966, pp. 87-112.

- "Técnicas de fiação primitivas: as rocas portuguesas", Cadernos de Etnografia, 2. ${ }^{a}$ série, 2, Barcelos, 1967, 38 pp., 50 figs.

- Escultura Africana no Museu de Etnologia do Ultramar, Lisboa, JIU, 1968 (em colaboração com Ernesto Veiga de Oliveira, Luís Polanah e Fernando Galhano).

- Construções Primitivas em Portugal, Lisboa, IAC, 1969, 363 pp., 459 figs. (em colaboração com Ernesto Veiga de Oliveira e Fernando Galhano).

- "Attrelage des boeufs au Portugal", Narodopisny Véstnik césc Kolovensky, III-IV, BrnoPraga, 1969, pp. 65-78 (em colaboração com Ernesto Veiga de Oliveira e Fernando Galhano).

- "O trajo popular e a tecelagem doméstica em Portugal”, Actas do XXIX Congresso Luso-Espanhol, III, Lisboa, Associação Portuguesa para a Progresso das Ciências, 1970, pp. 169-181.

- "Tecelagem manual e doméstica em Terroso", Boletim Cultural da Câmara Municipal da Póvoa de Varzim, X (2), Póvoa de Varzim, 1971, pp. 239-262, 9 figs.

- Internationale Volskskundliche Bibliographie, 1969-1970, Bona, Rudolf Habelt Verlag, 1972, 780 pp. (colaboração no que respeita à etnologia portuguesa).

- Museu de Etnologia do Ultramar: Povos e Culturas, Lisboa, JIU, 1972, 236 pp. 219 figs. (a colaboração corresponde a toda a documentação fotográfica e textos das legendas n. ${ }^{\circ} 2,78-81$ e 166-315).

- "Uma festa de iniciação das raparigas de Malembo - Cabinda", Geographica: Revista da Sociedade de Geografia de Lisboa, IX (33), Lisboa, 1973, pp. 29-50, 10 figs.

- Máscaras Portuguesas, Lisboa, JIU, 1973, 156 pp., 111 figs.

- Sistemas de Atrelagem dos Bois em Portugal, Lisboa, IAC, 1973, 124 pp., 197 figs. (em colaboração com Ernesto Veiga de Oliveira e Fernando Galhano).

- Internationale Volkskundliche Bibliographie, 1971-1972, Bona, Rudolf Habelt Verlag, 1974, 816 pp. (colaboração no que respeita à etnologia portuguesa).

- “Rio de Onor 1973”, em In Memoriam: António Jorge Dias, Lisboa, IAC, 1974, pp. 285 -305, 11 figs. (em colaboração com Ernesto Veiga de Oliveira e Fernando Galhano).

- Actividades Agro-Marítimas em Portugal, Lisboa, IAC, 1975, 236 pp., 216 figs. (em colaboração com Ernesto Veiga de Oliveira e Fernando Galhano).

- Alfaia Agrícola Portuguesa, Lisboa, IAC, 1976, 395 pp., 259 desenhos, 270 fotos, 3 mapas etnográficos (em colaboração com Ernesto Veiga de Oliveira e Fernando Galhano).

- Traje Popular, catálogo da exposição realizada pelo Museu de Etnologia no Museu Nacional do Traje, Lisboa, 1977, 46 pp., 78 figs., 36 desenhos.

- Escultura Africana, catálogo da exposição do Centro de Arte Contemporânea no Porto, 1977 (em colaboração com Ernesto Veiga de Oliveira).

- Tecnologia Tradicional Portuguesa: O Linho, Lisboa, INIC, 1978, 246 pp., 121 figs., 112 desenhos (em colaboração com Ernesto Veiga de Oliveira e Fernando Galhano).

- Internationale Volskskundliche Bibliographie, 1975-1976, Bona, Rudolf Habelt Verlag, 1979, 727 pp. (colaboração no que respeita à etnologia portuguesa).

- Internationale Volkskundliche Bibliographie, 1977-1978, Bona, Rudolf Habelt Verlag, 1981, 775 pp. (colaboração no que respeita à etnologia portuguesa).

- À Descoberta de Portugal, Lisboa, Selecções do Reader's Digest, 1982 (colaboração na parte etnográfica e artigo sobre "As máscaras transmontanas"). 
- "Les maures et les bugios de Sobrado (Valongo)", Les Cahiers Binchois: Revue de la Société d'Archéologie et des Amis du Musée de Binche, 5, Binche, 1982, pp. 34-44.

- Tecnologia Tradicional Portuguesa: Sistemas de Moagem, Lisboa, INIC, 1983, 520 pp., 491 figs., 246 desenhos (em colaboração com Ernesto Veiga de Oliveira e Fernando Galhano).

- Escultura Africana, legendas e fotografias do catálogo da exposição, Lisboa, Centro de Arte Moderna da Fundação Calouste Gulbenkian, 1985, pp. 19-42.

- “Máscaras transmontanas”, Brigantia: Revista de Cultura, V (2/3/4), Bragança, 1985, pp. 497-514.

- Internationale Volkskundliche Bibliographie, 1979-1980, Bona, Rudolf Habelt Verlag, 1985, 345 pp. (colaboração no que respeita à etnologia portuguesa).

- Têxteis: Tecnologia e Simbolismo, Lisboa, IICT, 1985, 99 pp., 38 figs.

- O Pão e o Bragal, Paredes de Coura, Câmara Municipal de Paredes de Coura, 1985, 36 pp., 12 figs. (em colaboração com Ernesto Veiga de Oliveira).

- "Máscaras do ciclo de Inverno em Trás-os-Montes", Atlantis, V (3), Lisboa, 1985, pp. 9-15.

- "A cultura material do povo português: uma visita ao Museu de Etnologia", em Reflexões sobre a História e Cultura Portuguesa: Temas de Cultura Portuguesa, 9, Lisboa, IPED, 1985, 261-270.

- "Migrações temporárias e estacionais: barrosões no Alentejo", em Estudos em Homenagem a Mariano Feio, Lisboa, Barbosa e Xavier, 1986, pp. 541-563 (em colaboração com Ernesto Veiga de Oliveira e Fernando Galhano).

- Máscaras Portuguesas, catálogo da exposição integrada na Bienal Universitária de Coimbra, Coimbra, ed. Museu de Etnologia, 1986, 26 pp.

- Internationale Volkskundliche Bibliographie, 1981-1982, Bona, Rudolf Habelt Verlag, 1986, 377 pp. (colaboração no que respeita à etnologia portuguesa).

- Tecnologia Tradicional Agrícola dos Açores, Lisboa, INIC, 1987, 94 pp., 146 figs., 43 desenhos (em colaboração com Ernesto Veiga de Oliveira).

- "Em defesa e para a valorização do património etno-museológico do Alto Minho", Mealibra: Revista de Cultura, 2. ${ }^{a}$ série, 1, Viana do Castelo, 1987, pp. 46-56.

- "La culture traditionelle", L'Europe aujourd'hui. Le Portugal, Bruxelas, Artis Historia, 1988, pp. 136-156.

- "Serração manual: parceria elementar e desigualdade", em Estudos e Ensaios em Homenagem a Vitorino Magalhães Godinho, Lisboa, Sá da Costa, 1988, pp. 507-514.

- "Regionalismo cultural: a propósito da reedição de Traje à Vianesa de Cláudio Basto", Cadernos Vianenses, XI, Viana do Castelo, 1988, pp. 57-63.

- "Ernesto Veiga de Oliveira e o Museu de Etnologia", em Estudos em Homenagem a Ernesto Veiga de Oliveira, Lisboa, INIC, 1989, pp. 555-568.

- Tecnologia Tradicional Portuguesa: Sistemas de Serração de Madeira, Lisboa, INIC, 1990, 160 pp., 92 figs., 44 desenhos.

- "Dados biográficos e autobiográficos de Ernesto Veiga de Oliveira”, Trabalhos de Antropologia e Etnologia, 30, "Homenagem a Ernesto Veiga de Oliveira", Porto, 1990, pp. 9-28.

- "Arcaísmo tecnológico: a extracção do azeite em dois lagares do Minho", Trabalhos de Antropologia e Etnologia, 30, "Homenagem a Ernesto Veiga de Oliveira", Porto, 1990, pp. 115-127. 
- "Entrevista com Sandra Xavier", em Olhares sobre Portugal: Cinema e Antropologia, Lisboa, CEAS, 1993, pp. 53-58.

- "Três filmes etnográficos sobre Portugal: comentários", em C.C. Boisvert (coord.), Ethnologie du Portugal: Unité et Diversité, Paris, Centre Culturel C. Gulbenkian, 1994, pp. 125-134.

- "A cozinha rural", em Vinte Valores do Mundo Rural, Lisboa, Instituto de Estruturas Agrárias e Desenvolvimento Rural, 1995, 3 pp.

- "Alfaias agrícolas", em O Voo do Arado, catálogo de exposição, Lisboa, Museu Nacional de Etnologia, 1996, pp. 161-198.

- "Fertilizantes naturais", em O Voo do Arado, catálogo de exposição, Lisboa, Museu Nacional de Etnologia, 1996, pp. 201-214.

- "A luz da memória: Benjamim Enes Pereira e os caminhos da antropologia em Portugal", entrevista de Paula Godinho, Arquivos da Memória, 1, Lisboa, CEEP, 1996, pp. 69-73.

- "Falares entretecidos", em O Fio da História , catálogo de exposição, Museu Municipal de Loures, 1996, pp. 5-19 (em colaboração com Luís Vasconcelos).

- Evocação do Mundo Agrícola de Paredes de Coura, Paredes de Coura, 1997, 68 pp., 12 figs. (em colaboração com Joana Rodrigues).

- Tecnologia Tradicional do Azeite em Portugal, Idanha-a-Nova, Centro Cultural Raiano, 1997, 159 pp., 107 figs., 3 desenhos.

- Escultura Tchokwe, Marie-Louise Bastin, catálogo de exposição, Fundação Cupertino de Miranda, Faculdade de Letras da Universidade do Porto, 1999, 153 pp., 54 figs. (concepção de exposição e catálogo).

- "Relato acerca da organização da colecção dos instrumentos musicais populares portugueses", em Ernesto Veiga de Oliveira, Instrumentos Populares Portugueses, Lisboa, Fundação Calouste Gulbenkian/Museu Nacional de Etnologia, 2000, pp. 11-33.

- Sociedade Rural do Barroso, Museu Rural de Boticas, Boticas, 2000, pp. 21-70, 29 figs., 13 desenhos.

- "Moinhos de vento de Montedor", em Festas em honra de Nossa Senhora da Graça, Carreço, Junta de Freguesia de Carreço, 2001, 11 pp., 5 figs., 6 desenhos.

- "Recordando o fabrico caseiro de manteiga", XVII Festival de Folclore, Grupo Folclórico e Cultural de Danças e Cantares de Carreço, Agosto de 2001 (2 páginas, não numeradas).

- "Máscaras portuguesas", em Máscaras em Portugal, Lisboa, Mediatexto, 2003, pp. 8-15.

- "Museu da Luz", em Museu da Luz, catálogo de exposição, Luz, Museu da Luz/EDIA, 2003, pp. 47-83 e 123-152.

- "Museu da Luz", Etnográfica, VII (1), 2003, pp. 209-212.

- O Linho é um Sonho, Castelo Branco, Museu Francisco Tavares Proença Júnior, 2003, 28 pp., 21 figs.

- A Seda é um Mistério, Castelo Branco, Museu Francisco Tavares Proença Júnior, 2003, 20 pp., 17 figs.

- A Lã e o Linho no Traje do Alto Minho, Viana do Castelo, Câmara Municipal de Viana do Castelo, 2004, 44 pp., 57 figs.

- Testemunhos da Arquitectura Tradicional: Monte dos Pássaros, Futuro Núcleo do Museu da Luz, Textos Exposição, Luz, Museu da Luz/EDIA, 2005.

- Rituais de Inverno com Máscaras, coordenação de catálogo de exposição, Bragança, Museu Abade de Baçal/IPM, 2006. 
- "Rituais de Inverno com máscara", em Rituais de Inverno com Máscaras, Bragança, Museu Abade de Baçal/IPM, 2006, pp. 13-37.

- "Terras de centeio e castanha no Norte de Portugal", em Arte e Cultura de Galicia e Norte de Portugal: Etnografia, Tomo II, Nova Galicia Ediciones, 2006, pp. 62-102 (em colaboração com Clara Saraiva).

- "Conversas à mesa da memória do Museu da Luz", em Olhar o Monte Alentejano a Pretexto de Alqueva, Museu da Luz, EDIA, 2007, pp. 123-131.

- "O traje dito à vianesa", em Uma Imagem da Nação: Traje à Vianesa, Viana do Castelo, Câmara Municipal de Viana do Castelo, 2009, pp. 117-215, 100 figs., 14 desenhos (em colaboração com António Medeiros e João Alpuim).

\section{ACTIVIDADE CIENTÍFICA}

- Membro da equipa fundadora do Museu de Etnologia (com Jorge Dias, Margot Dias, Ernesto Veiga de Oliveira e Fernando Galhano), Lisboa.

- Investigador do Centro de Estudos de Etnologia (CEE), Instituto Nacional de Investigação Científica (INIC).

- Investigador do Centro de Antropologia Cultural e Social (CACS), Instituto de Investigação Científica Tropical (IICT).

- Encarregado de missão, Museu de Etnologia, Instituto de Investigação Científica Tropical (IICT).

\section{Recolha de materiais etnográficos para o Museu de Etnologia}

- Portugal continental e insular:

Alfaia agrícola;

Transportes;

Tecnologias e produtos artesanais;

Instrumentos musicais;

Trajes e têxteis;

Utensilagem agro-marítima;

Arte pastoril;

Iluminação;

Utensilagem de cozinha.

- Angola:

Máscaras ligadas à sociedade Zindunga, de Cabinda;

Instrumentos musicais;

Celeiros construídos em palha;

Utensilagem doméstica;

Esculturas.

\section{Organização sistemática das colecções do Museu de Etnologia}

- Formulação do critério seguido para a organização do ficheiro sistemático, segundo a classificação de P. Murdock (Outline of Cultural Materials), adaptado à temática museológica.

- Direcção da arrumação sistemática das reservas do Museu de Etnologia. 
- Organização das reservas do Museu de Etnologia respeitantes ao mundo rural português para a sua abertura ao público sob a forma de "Galerias do Mundo Rural".

\section{Coordenação e montagem de exposições}

- "Instrumentos Musicais Portugueses", Fundação Calouste Gulbenkian, Lisboa, 1962.

- "Alfaia Agrícola Portuguesa", ISCSPU, Lisboa, 1968.

- "Povos e Culturas", Galeria Nacional de Arte Moderna, Lisboa, 1972.

- "Modernismo e Arte Negro-Africana”, Museu de Etnologia, Lisboa, 1976.

- "Arte Africana", Centro de Arte Contemporânea, Porto, 1977.

- “Trás-os-Montes: A Mão do Homem”, Vila Real, 1982.

- "Alfaia Agrícola Portuguesa", Coimbra, 1983.

- "Máscaras Transmontanas", Chaves, 1984.

- “Cultura e Tradição: Guiné-Bissau”, Porto, 1984.

- “Instrumentos Musicais Populares Portugueses”, Coimbra, 1985.

- "Escultura Africana em Portugal", Lisboa, 1985.

- “Têxteis: Tecnologia e Simbolismo", Lisboa, 1985.

- "Desenho Etnográfico de Fernando Galhano", Lisboa, Porto, Coimbra, Figueira da Foz, 1985/1987.

- “Máscaras Portuguesas", Coimbra, 1986.

- "Instrumentos Musicais Populares Portugueses", Porto, 1986.

- "Trabalho e Festa nas Lavouras do Noroeste", Ponte de Lima, 1986.

- “Máscaras de Trás-os-Montes”, Sappada, Itália, 1986.

- "Os Instrumentos Musicais e as Viagens dos Portugueses", Lisboa, 1986.

- “Índios da Amazónia”, Museu de Etnologia, Lisboa, 1986.

- “Angola: Povos e Culturas", Museu de Etnologia, Lisboa, 1987.

- “Olaria Portuguesa Decorada”, Vila Nova de Cerveira, 1987.

- “Timor de Ruy Cinatti”, Lisboa, 1988.

- "Povos de Timor, Povo de Timor: Vida, Aliança e Morte", Museu de Etnologia, Lisboa, 1990.

- "Um Olhar sobre Macau", Museu de Etnologia, Lisboa, 1990.

- "O Fado", Museu de Etnologia, Lisboa, 1994.

- "Goa", Museu de Etnologia, Lisboa 1995.

- "O Voo do Arado", Museu de Etnologia, Lisboa, 1996.

- Centro Cultural Raiano, Idanha-a-Nova, Castelo Branco, 1997.

- Museu Regional de Paredes de Coura, 1997.

- Museu da Terra e do Mar, Carrapateira, 1999/2000.

- Organização das “Galerias do Mundo Rural”, Museu Nacional de Etnologia, 2000.

- Museu Rural de Boticas, Montalegre, 2000.

- Museu Tavares Proença, Castelo Branco, 2002.

- Sala de exposições temporárias e "Sala da Memória”, Museu da Luz, 2003.

- Exposição “Ein Dorf zieht um”, Museum fur Volkerkunde, Hamburgo, 2004 (em colaboração com Clara Saraiva). 
- "Homenagem a Fernando Galhano", Porto, 2004.

- Museu do Traje, Viana do Castelo, 2004.

- Museu do Canteiro, Alcains, 2005.

- "Memórias e Saberes", Núcleo Expositivo de Lagares da Beira, 2006.

- "Rituais de Inverno com Máscaras", Museu Abade de Baçal, Bragança, 2006.

- Museu Agro-Marítimo de Carreço, Núcleo Museológico do Museu do Traje de Viana do Castelo, 2008.

\section{Realização e coordenação científica de documentários e filmes etnográficos e outros materiais audiovisuais}

Realização de documentários cinematográficos, em $16 \mathrm{~mm}$, sobre temas etnográficos

- A Apanha do Sargaço no Norte de Portugal;

- Cestaria de Vime (Ílhavo);

- Olaria em Malhada Sorda;

- Olaria de Campo Maior;

- Olaria de Asseiceira;

- Debulha de Cereal a Trilho (Terras de Miranda);

- Uma Malha de Centeio (a Manjual) em Terras de Basto;

- Danças Tradicionais Integradas na Festa da Senhora dos Altos Céus, na Lousa;

- Uma Vessada no Alto Minho (Paredes de Coura);

- Pastoreio no Barroso;

- Uma Lavra em Buços (Cabeceiras de Basto);

- Moinho de Vento Alentejano, Almodôvar;

- Santa Vitória do Ameixial: Festas e Bênção do Gado;

- Tear de Esteiras, Alcácer do Sal;

- Tear de Esteiras, Arzila, Coimbra;

- Tear Vertical - S. Brás de Alportel;

- Fabrico de Crossas - Deão, Viana do Castelo;

- Ciclo do Linho: A Planta - Fornelos, Ponte de Lima;

- Ciclo do Linho: O Fio;

- Ciclo do Linho: O Tecido;

- Cestaria de Madeira Rachada - Tecla, Celorico de Basto;

- Engenho de Serrar Madeira de Tracção Hidráulica - Gemeses, Esposende;

- Engenho do Linho de Tracção Animal - Penafiel.

\section{Orientação científica de filmes e documentários etnográficos}

Documentários cinematográficos realizados em Portugal sob a orientação científica de Benjamim Pereira, em colaboração com Ernesto Veiga de Oliveira, pelo Institut für den Wissenschaftlichen Film Gottingen (Instituto do Filme Científico de Gottingen), em 1970:

- Fischen von Wasserpilanzen in der Ria de Aveiro;

- Tangfischerei bei Castelo de Neiva; 
- Brotbacken in Perafita;

- Fischerei mit dem, Zugnetz in Torreira;

- Dreschen und Dreschbau in Tecla;

- Topfern von krugen in Malhada Sorda;

- Brennen von topware in Malhada Sorda;

- Stockspiel in Basto;

- Tochwalken in Tabuadela;

- Stierkampf in Forcalhos;

- Jahrmarkt und Stierkampf in Cuba;

- Kirchfest «Romaria » von Salvador do Mundo;

- Kirchfest «Romaria » von S. Bartolomeu do Mar;

- Kirchfest «Romaria » von S. João d’Arga.

Coordenação Científica de outros filmes etnográficos, realizados no âmbito de pesquisas de terreno para museus e centros culturais locais e regionais:

- O Linho é um Sonho, realização de Catarina Alves Costa, 2003, Museu de Francisco Tavares Proença Júnior, Castelo Branco;

- A Seda é um Mistério, realização de Catarina Alves Costa, 2003, Museu de Francisco Tavares Proença Júnior, Castelo Branco;

- Acervo Audiovisual, Museu da Luz, Mourão, Évora, 2003-2004;

- Rituais de Inverno com Máscara, Bragança, Museu Abade de Baçal, 2006.

Recolhas de material fotográfico que fazem parte dos arquivos do Centro de Estudos de Etnologia (CEE), do Centro de Antropologia Cultural e Social (CACS/IICT) e do Museu de Etnologia:

- 8000 negativos P/B e cor - Angola;

- 20.000 negativos P/B e cor - Portugal, Açores e Madeira.

\section{Outras actividades}

- Recolha e organização da documentação representativa de Portugal no Museu Internacional do Carnaval e da Máscara de Binche, Bélgica.

- Análises e peritagens de arte africana.

- Apoio a licenciandos em antropologia cultural. 